
\title{
$\checkmark$ Research Square \\ Local injection of anti-cancer drugs in an experimental model of colorectal cancer, a novel scenario to treat invasive neoplasia.
}

Vicente Lorenzo-Zúñiga ( $\nabla$ vlorenzozuniga@gmail.com )

Hospital Universitari i Politecnic La Fe https://orcid.org/0000-0002-4072-3241

Ignacio Bon

Foundation Institute of Research in Health Sciences Germans Trias i Pujol: Fundacio Institut d'Investigacio en Ciencies de la Salut Germans Trias i Pujol

Napoleón de la Ossa

Hospital Germans Trias i Pujol: Hospital Universitari Germans Trias i Pujol

\section{Ramón Bartolí}

Foundation Institute of Research in Health Sciences Germans Trias i Pujol: Fundacio Institut d'Investigacio en Ciencies de la Salut Germans Trias i Pujol

\section{Research Article}

Keywords: colorectal cancer, drug-eluting platform, intratumoral injection, experimental model

Posted Date: June 17th, 2021

DOl: https://doi.org/10.21203/rs.3.rs-632509/v1

License: (c) (1) This work is licensed under a Creative Commons Attribution 4.0 International License. Read Full License 


\section{Abstract}

Purpose: Endoscopic resection of colorectal lesions significantly decreases the risk of colorectal cancer (CRC) incidence and death. Patients with invasive neoplasia or metastatic CRC have an evident poorer prognosis. Next step could be a more personalized treatment strategy, as a targeted therapy by direct injection of active substances in a selected lesion. The aim was to evaluate the efficacy of intratumoral injection of a drug-eluting platform (CoverGelâ) with different anti-cancer drugs in a rat model of azoxymethane (AOM)-induced CRC.

Methods: Experimental CRC was induced in 20 rats. Animals were randomized in five groups (four each): CoverGel + Aflibercept (AFL), CoverGel + Bevacizumab (BVZ), CoverGel + Cetuximab (CTX), CoverGel + Panitumumab (PNT) and CoverGel + Irinotecan (IRT). Endoscopic, histological and necrosis score (0-3 scale) test were performed during follow-up to evaluate clinical success.

Results: All animals developed invasive neoplasia (NICE 3, JNET 3), $7.2 \mathrm{~mm}$ as mean size (range 6-8). Intratumoral injection was feasible in all animals with no adverse events. AFL, an angiogenesis inhibitor agent, in comparison with the other medications (BVZ, CTX, PNT and IRT) obtained the best results: significantly reduction in tumor size $(-30 \%$ vs. $-10 \%,-3 \%,+2 \%$ and $+2 \% ; p<0.05$, respectively) and significantly higher necrosis score ( 3 vs. $1,0.3,0$ and $0 ; p<0.05$, respectively).

Conclusion: Angiogenesis inhibitors medications produced the higher cell necrosis and reduction in tumor size in an experimental model of CRC. Intratumoral injection of a drug-eluting platform could open a new way to manage invasive CRC.

\section{Introduction}

Colonoscopic procedures have become massive in the last years since colorectal cancer (CRC) is becoming a prevalent disorder, with over 450,000 new cases / year in Europe $(1,2)$. Despite declines in recent decades, one-third of patients with CRC surviving less than 5 years (3). The current guidelines recommend surgical resection for nonmetastatic T1 CRC tumors or high-risk polyps, but most of these neoplasms could be safely and effectively removed with endoscopic resection techniques (EMR, ESD, EFTR) (4-6). Moreover, recent data confirmed endoscopic therapy has the highest quality-adjusted-lifeyear and the lowest cost for T1 CRC (7). Next step in the treatment of this molecularly complex disease could develop more personalized treatment strategies to identify the best candidates and further lower the CRC mortality rate. In this sense, a targeted therapy to provide an active substance in a precise site of the colon has many advantages to achieve the optimal therapy for different classes of patients by facilitating dose adjustment and limiting side effects. The development of drug-eluting platforms with triple biologic activity (TriBio; Bioadhesive-Biodegradable-Bioactive) that can be applied directly on the lesions to be treated has opened a new line of research, as has been demonstrated by our group in inflammatory bowel disease (8). Following these evidences we have tested our TriBio platform 
(CoverGel $\rightarrow$ ) to evaluate the efficacy of intratumoral injection with different combinations of antitumoral drugs in a rat model of azoxymethane-induced CRC.

\section{Materials And Methods}

\section{Drug-eluting platform}

We used a patented combination of hyaluronic acid, methylcellulose and Poloxamer Pluronic-F127 (WO 2018/019881 A1). This composition showed a good biocompatibility in in vitro and in vivo studies, and drug release following a Fickian diffusion curve, related to the concentration of the drug in the medium (8).

\section{Endoscopy}

All endoscopic evaluations were carried out using an Olympus FQ260Z (Olympus, Tokyo, Japan), with an outer diameter of $9.5 \mathrm{~mm}$, working channel diameter of $2.8 \mathrm{~mm}$. Room air was used for insufflation during the endoscopy. Endoscopic injection of TriBio platform was done through an injection needle 21G.

\section{Colon Carcinogenesis Animal Study}

CRC was induced by two s/c applications of azoxymethane (AOM, $15 \mathrm{mg} / \mathrm{kg}$ ) given one week apart (9). Twenty male Sprague-Dawley rats (Harlan Laboratories Models SL, Barcelona, Spain) weighing 100$125 \mathrm{~g}$ were included in this study. Animals were followed during 35 weeks to develop colonic tumors. Animals were randomized at day 0 in five groups treated with intratumoral injection of CoverGel $\rightarrow$ with different combinations of anti-vascular endothelial growth factor (Anti-VEGF; Aflibercept -AFL-, Bevacizumab -BVZ-), anti-epidermal growth factor receptor (Anti-EGFR; Cetuximab -CTX-, Panitumumab -PNT-) or cytotoxic topoisomerase inhibitor (Irinotecan -IRT-) drugs: (i) CoverGel + AFL (2 mg/mL) group, (ii): CoverGel + CTX (16 mg/mL) group, (iii): CoverGel + PNT (6 mg/mL) group, (iv): CoverGel + IRT (3.5 mg/mL) group, and (v): CoverGel + BVZ $(5 \mathrm{mg} / \mathrm{mL})$ group. A total amount of $0.5 \mathrm{~mL}$ of solution was applied in all groups; thus, the dosage of the drug applied to groups were $1 \mathrm{mg}$ of AFL, $8 \mathrm{mg}$ of CTX, $3 \mathrm{mg}$ of PNT, $1.7 \mathrm{mg}$ of IRT and $2.5 \mathrm{mg}$ of BVZ, respectively.

Animals underwent colonoscopy on days 0 and 10 to describe lesions. Ten days after treatment (Day 10), animals were euthanatized by an anesthetic overdose. Total colon was evaluated for macroscopic \& histological tumor study: incidence, multiplicity, localization (proximal /distal), classification, size (Image $\mathrm{J}$ software) and necrosis score (0-3 scale: absent, mild, moderate, severe) (Fig. 1). Treated site samples were cut and placed in formaldehyde for $\mathrm{H} / \mathrm{E}$ staining and blinded histopathological evaluation.

\section{Statistical Study}

All values are expressed in median \pm range unless otherwise stated. The comparison of groups was done using two-way ANOVA comparison test as a post hoc.

\section{Animal Care}


All animals were obtained from ENVIGO. All protocols were by the Animal Experimentation Ethics Committee of the Hospital Universitari Germans Trias I Pujol (registered as B9900005) and approved by the Departament d'Agricultura, Ramaderia, Pesca, Alimentació i Medi Natural of the Catalan Regional Government (protocols number 8,585 and 10,235) according to current national and European Union legislation regarding the protection of experimental animals. Rats were supervised daily in order to ensure animal welfare and euthanized, if required, by anesthetization with isoflurane. All animals had free access to water and food ad libitum. Animals were monitored daily after any procedure, and $200 \mathrm{mg} / \mathrm{kg}$ of paracetamol was administered intraperitoneally. Normal parameters of well being such as hair health and secretions were analyzed.

\section{Results}

\section{Colorectal Epithelial Lesions}

After 35 weeks of AOM application all animals developed distal $(n=20)$ with/without proximal $(n=5)$ invasive malignant colonic tumors (NICE Type 3, JNET Type 3). Mean number of lesions was 1.25 (range $1-2$ ), with a mean size of $7.2 \mathrm{~mm}$ (range 6-8).

\section{Efficacy of TriBio platform}

Endoscopic, macroscopic and histological evaluations are showed in Fig. 3. Anti-VEGF agents (AFL and BVZ) showed higher efficacy as compared with the other drugs. Tumor size reduction after administration of different drugs was significantly higher with AFL $(-30 \%)$ vs. the others (BVZ $-10 \%$, PNT $+2 \%, C T X-$ $3 \%, I R T+2 \%) ; p<0.05$ vs. all others respectively. Tumor necrosis score was also significantly higher with AFL vs. the others ( 3 vs. 1, 0.3, 0 and 0$) ; p<0.05$ vs. all others respectively. PNT and IRT were ineffective in locally treat these lesions (Fig. 4).

\section{Discussion}

In this paper we have reported the application of a drug-eluting hydrogel with proven biocompatible, bioadhesive and bioactive properties to perform endoscopic treatment of invasive colorectal tumors. Endoscopic resection of precancerous colorectal lesions is one the most frequently performed medical interventions, which significantly decreases the risk of colorectal cancer incidence and death. However, patients with invasive neoplasia or metastatic CRC have to undergo surgical resection or chemotherapy treatment, with an evident poorer prognosis. In this sense, a targeted treatment to administrate an antitumoral agent in an advanced tumor could improve the mortality rate in these cases. Local injection of bioactive agents is a novel scenario for endoscopic therapy that has been proposed by our group in experimental and in clinical practice $(8,10)$.

AOM colon carcinogenesis is a multistep process with morphological and histological features similar to those seen in human sporadic carcinogenesis $(11,12)$. In this process dysplastic aberrant crypts progress to advances adenomas and into adenocarcinomas. 
Cytotoxic (IRT) or molecular targeted medications (anti-EGFR or anti-VEGF) are commonly used in the systemic therapy to prevent postoperative recurrence or to treat unresectable colorectal cancer (13). These drugs can induce different side effects, by affecting the angiogenesis (anti-VEGF) or avoiding cells grow (anti-EGFR). Targeted drugs work with the same mechanism of action, at a minor dosage, and less side effects. Tumor angiogenesis is the main factor for tumor growth and metastasis (14). Growth at the primary tumor site, local tissue infiltration and distant metastasis of CRC have been demonstrated to be highly dependent on angiogenic vessel formation, where VEGF is the key angiogenic factor (15). In our study demonstrated the feasibility to applicate an intratumoral injection of a drug-eluting platform with anti-cancer agents, and than vessel inhibition, as compared with growth inhibition, is more important in the earlier stage of tumorigenesis and progression. AFL and BVZ are anti-VEGF agents with different targets, $B V Z$ is a monoclonal Ig $G$ antibody against VEGF-A, and AFL is a fusion protein with high VEGF

affinity (VEGF-A and VEGF-B). Our results confirmed than AFL, induced the highest reduction in tumor size and necrosis in our experimental model of CRC.

In conclusion, and recognizing that animal models do not represent the complexity of the human disease and do not replace clinical studies, these evidences could open a new to manage patients with unresectable or metastatic CRC to prevent recurrence or progression.

\section{Declarations}

Funding: This work was supported by project PI13/02217 given to VL-Z by the Health Institute Carlos III www.isciii.es

Conflict of interest: Vicente Lorenzo-Zúñiga and Ramon Bartoli are the authors of the patent (WO 2018/019881 A1)

\section{References}

1. Zauber AG, Winawer SJ, O'Brien MJ, et al Colonoscopic polypectomy and long-term prevention of colorectal-cancer deaths. N Eng J Med 2012; 366(8): 687-96.

2. Gini A, Jansen EEL, Zielonke N, et al. Impact of colorectal cancer screening on cancer-specific mortality in Europe: A systematic review. Eur J Cancer 2020;127:224-235.

3. National Cancer Institute; Surveillance, Epidemiology, and End Results Program. Cancer stat facts: colorectal cancer. https://seer.cancer.gov/statfacts/html/colorect.html. Accessed December 10, 2019.

4. Raju GS, Lum PJ, RossWA, et al. Outcome of EMR as an alternative to surgery in patients with complex colon polyps. Gastrointest Endosc 2016;84(2):315-325.

5. Peery AF, Shaheen NJ, Cools KS, et al. Morbidity and mortality after surgery for nonmalignant colorectal polyps. Gastrointest Endosc 2018;87(1):243-250.e2. 
6. Rex DK, Hassan C, Dewitt JM. Colorectal endoscopic submucosal dissection in the United States: why do we hear so much about it and do so little of it? Gastrointest Endosc 2017;85(3):554-558.

7. Jang SR, Truong H, Oh A, Choi J, Tramontano AC, Laszkowska M, Hur C. Costeffectiveness Evaluation of Targeted Surgical and Endoscopic Therapies for Early Colorectal Adenocarcinoma Based on Biomarker Profiles. JAMA Netw Open 2020; 3(3):e1919963.

8. Bon I, Cano-Sarabia M, De la Ossa N, Bartolí R, Lorenzo-Zúñiga V. Development and characterization of a new endoscopic drug eluting platform with proven efficacy in acute and chronic experimental colitis. Frontiers in Medicine 2020 (in press).

9. Perse $\mathrm{M}$, Cerar A. Morphological and molecular alterations in 1,2 dimethylhydrazine and azoxymethane induced colon carcinogénesis in rats. J Biomed Biotechnol 2011; 2011: 474964.

10. Lorenzo-Zúñiga V, Boix J, Mañosa M, Lezcano C, Cabré E, Domènech E. Local injection of infliximab in symptomatic isolated mucosal lesions: A novel scenario for endoscopic therapy?. Inflamm Bowel Dis 2013; 19(4): E59-61.

11. Bird RP. Role of aberrant crypt foci in understanding the pathogenesis of colon cancer. Cancer Letters 1995; 93(1): 55-71.

12. Tanaka T. Colorectal carcinogenesis: review of human and experimental animal studies, Journal of Carcinogenesis 2009; 8: 5.

13. Hashiguchi Y, Muro K, Saito Y, et al. Japanese Society for Cancer of the Colon and Rectum (JSCCR) guidelines 2019 for the treatment of colorectal cancer. Int J Clin Oncol 2020; 25:1-42.

14. Hanahan D, Weinberg RA. Hallmarks of cancer: the next generation. Cell 2011; 144:646-674.

15. Seeber A, Gunsilius E, Gastl G, Pircher A. Anti-angiogenics: Their value in colorectal cancer therapy. Oncol Res Treat 2018; 41(4):188-193.

\section{Figures}




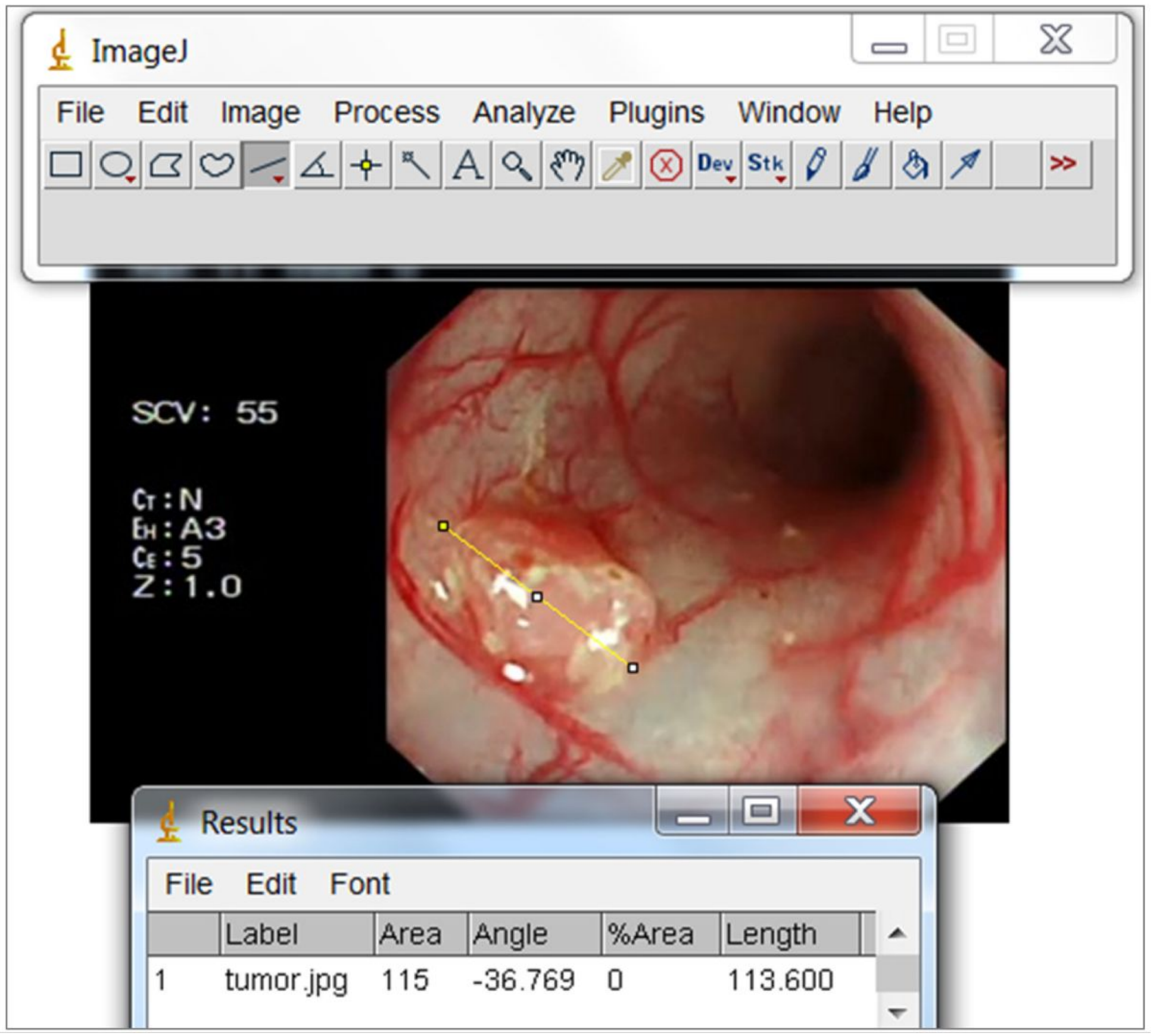

Figure 1

Tumor size measurement using free software (Image $\mathrm{J}$ ) 


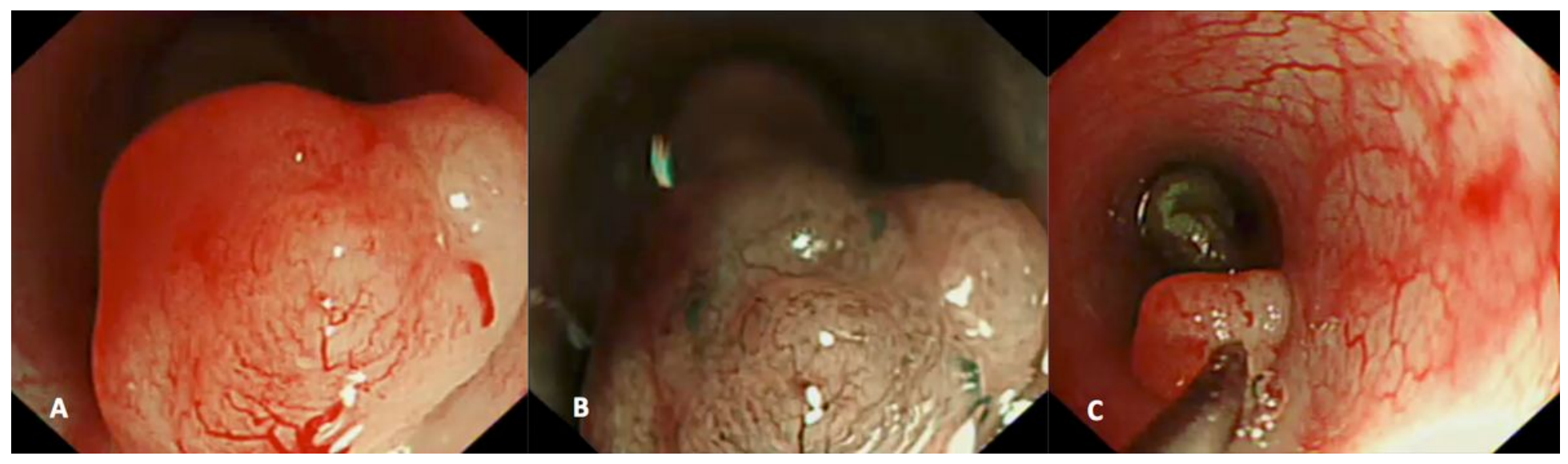

\section{Figure 2}

Endoscopic view of invasive colonic tumors: (a) White light; (b) NBI; (c) Intratumoral injection of drugeluting hydrogel 


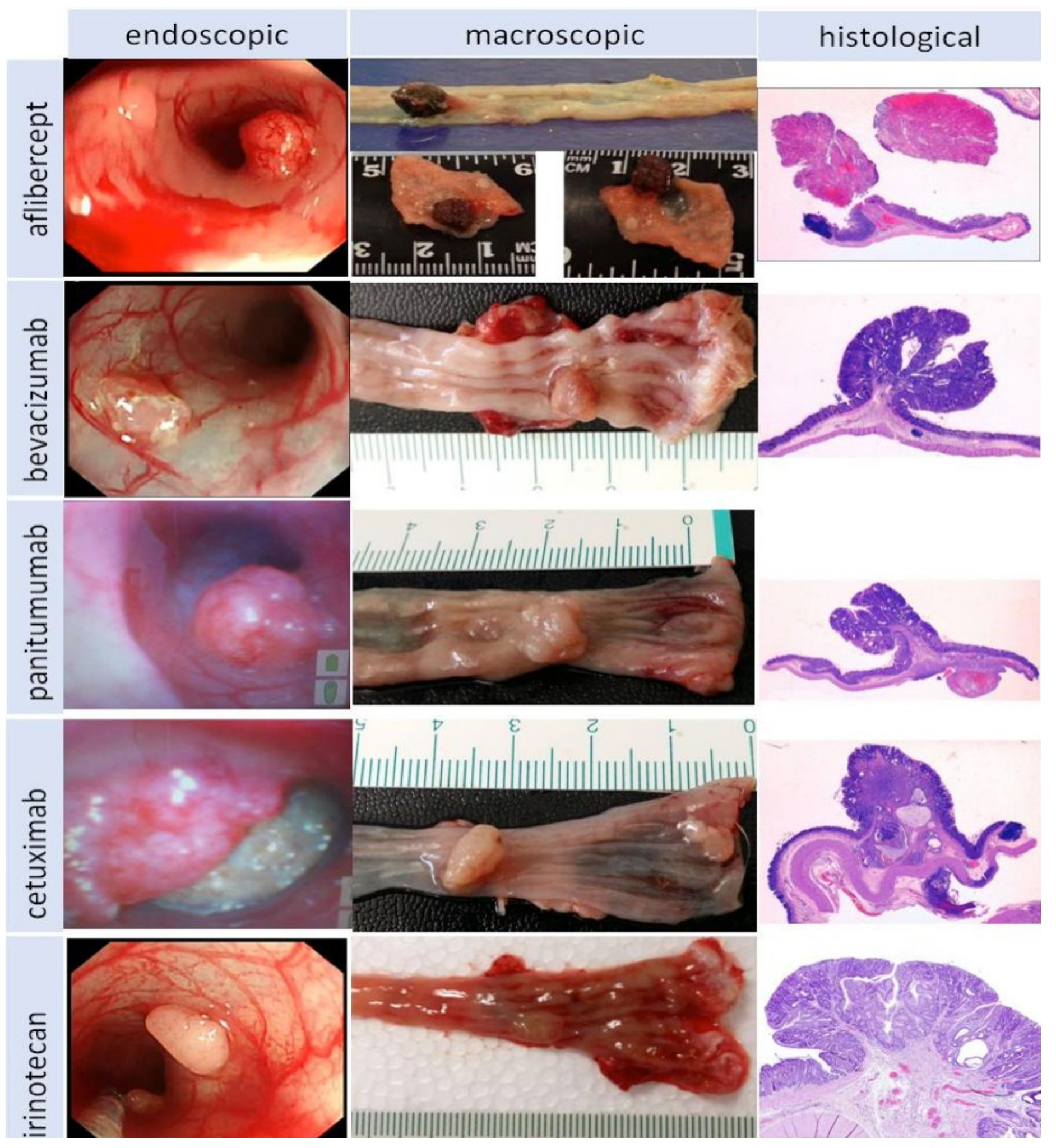

Figure 3

Endoscopic, macroscopic and histological evaluations of treated animals 

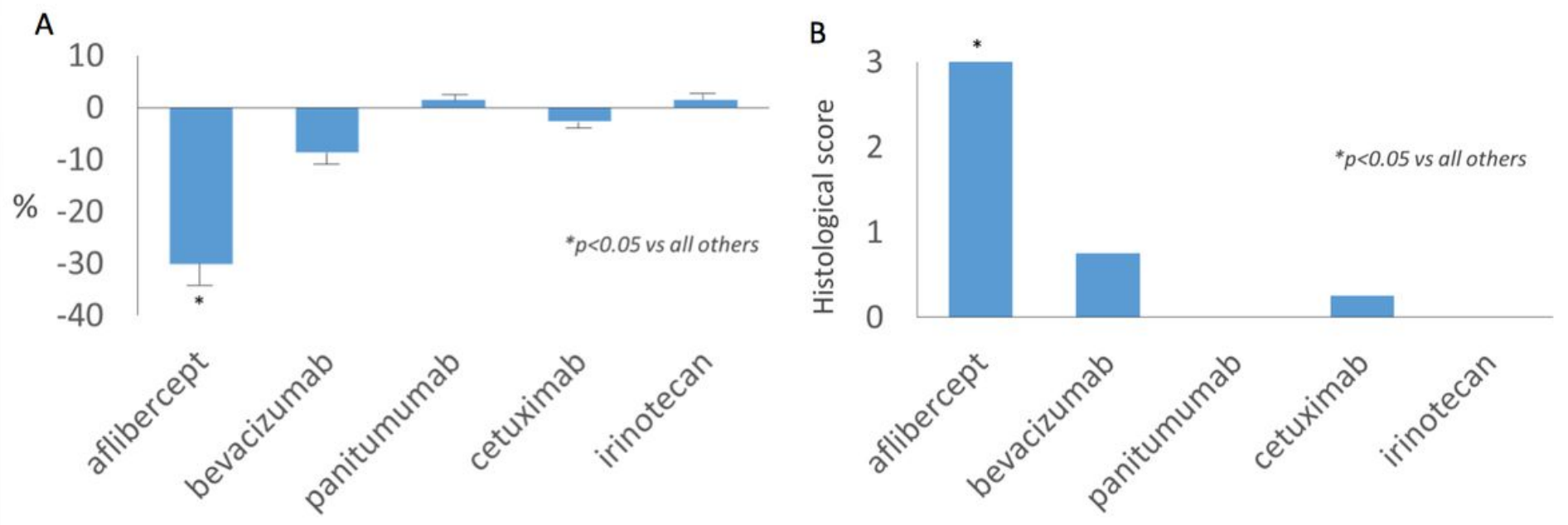

Figure 4

Efficacy after administration of different drugs: (a) Tumor size reduction; (b): Histological score of necrosis. 